\title{
Prognosis and Outcomes of Pneumolabyrinth Following an Otic- Capsule Violating Fracture: A Case Report
}

\section{Case Report}

\author{
Norah Alajmi', Abdullah Arafat ${ }^{2}$, Yazeed Aloqaili ${ }^{2}$ \\ ${ }^{1}$ College of Medicine, King Saud Bin Abdulaziz University, ${ }^{2}$ Otolaryngology Head \& Neck \\ Surgery Department King Abdulaziz Medical City, Saudi Arabia
}

\begin{abstract}
Background: Pneumolabyrinth consequent to a temporal bone fracture is a rare entity. It occurs when air enters the vestibulocochlear system due to a pathological communication between the middle and the inner ears. The diagnosis is usually confirmed radiologically using high-resolution computed tomography imaging. Pneumolabyrinth is categorized radiologically as follows: vestibular pneumolabyrinth, cochlear pneumolabyrinth and combined pneumolabyrinth.

Case Presentation: We herein describe the clinical presentation and management of pneumolabyrinth in a 43-year-old man, following an otic capsule-violating temporal bone fracture. After initially caring for the patient conservatively, without improvement, surgical intervention was undertaken. Unfortunately, hearing was irrecoverable.

Conclusion: Delaying medical and surgical intervention and the location of the air bubbles associated with the combined type contribute to the irrecoverable loss of hearing. As otic capsule-violating fractures might not be observed initially on brain CT scans, high-resolution and thin-slice CT scans of the temporal bone is crucial when suspecting temporal bone fracture.
\end{abstract}

Key Words: Hearing loss, otic capsule fracture, pneumolabyrinth, vestibular symptoms

Received: 2 December 2018, Accepted: 31 January 2019

Corresponding Author: Norah Alajmi, MD, College of Medicine, King Saud bin Abdulaziz University for Health Sciences, Kingdom of Saudi Arabia Tel.: +966553330542, E-mail: alajmin@outlook.sa

ISSN: 2090-0740, March 2019 Vol.20, No.1

\section{INTRODUCTION:}

Pneumolabyrinth is defined as the presence of air within the vestibule, semicircular canals, or cochlea, resulting from a pathological communication between the middle ear and the inner ear ${ }^{[1-2]}$ The presence of pneumolabyrinth on computerized tomography (CT) is highly suggestive a perilymphatic fistula. The radiological features suggestive of pneumolabyrinth was first described in 1984 by Mafee et al., ${ }^{[3]}$ while pneumolabyrinth secondary to a temporal bone fracture was first described in 1988 by Nurre et al ${ }^{[4]}$ Pneumolabyrinth is a rare entity with 50 cases reported in the literature, 15 of which were combined pneumolabyrinth secondary to temporal bone fracture ${ }^{[1-8]}$

Pneumolabyrinth can result from a temporal bone fracture, luxation of the stapes into the vestibule, after stapedectomy, or can be caused by barotrauma ${ }^{[1,4,5,6]}$ Symptoms include sensorineural hearing loss (SNHL), tinnitus, a sense of aural fullness, and dizziness. Highresolution, thin-slice CT of the temporal bone is recognized as the most appropriate imaging study to diagnose pneumolabyrinth.

A pneumolabyrinth can be categorized radiologically as follows: vestibular pneumolabyrinth, when air is confined to the vestibule and/or the semicircular canals (SCCs); cochlear pneumolabyrinth, when air bubbles are only detected in the cochlea; and combined pneumolabyrinth, when air is detected in both the vestibular organs and the cochlea ${ }^{[1]}$ Pneumolabyrinth is managed either conservatively or by surgical intervention.

We report the case of a 43-year-old man with radiologically confirmed right-sided combined pneumolabyrinth, secondary to a temporal bone fracture caused by a fall. Prognostic factors found in the current literature were compared to our patient. A review of literature is also presented to familiarize physicians with the prognostic factors of this unusual complication.

\section{Case}

Ethical approval was obtained to present this study and informed consent was provided by the patient. The 43 -yearold gentleman fell on the right side of his head while skating 3 weeks prior to his visit to the Otology clinic. He lost consciousness briefly after the fall. He also reported a history of a sense of fullness in the right ear; hearing loss; tinnitus; intense vertigo upon standing, bending forward, or quick turns of the head, and experiencing veering to the side while walking. The patient was otherwise healthy, with no history of previous problems of the ears, hearing, or balance. 
The patient initially presented to the emergency department and from there, he was referred to the Otology clinic. At his initial clinical evaluation there was no evidence of any neurological deficit. Otological examination revealed intact tympanic membranes with mild erythema of the right tympanic membrane. There was no facial asymmetry. The Weber test demonstrated lateralization to the left side and Rinne's test showed positive results on the left side. There was no spontaneous or gaze-evoked nystagmus.

A brain CT scan was performed in the emergency room and showed a small left temporo-parietal extra-axial hemorrhage. The same CT scan showed a non-depressed fracture of the squamous portion of the right temporal bone. There was no evidence of any fractures in the mastoid, tympanic, or petrous portions of the temporal bone. There was no evidence of any fractures violating the otic capsule. Small foci of air density were detected by CT scan within the middle turn of the cochlea and the vestibule, consistent with combined pneumolabyrinth (Figure 3). A pure tone audiogram (PTA) demonstrated right-sided, profound sensorineural hearing loss (Figure 1). Auditory evoked brainstem responses, electrocochleography, videonystagmography and caloric testing were not performed.

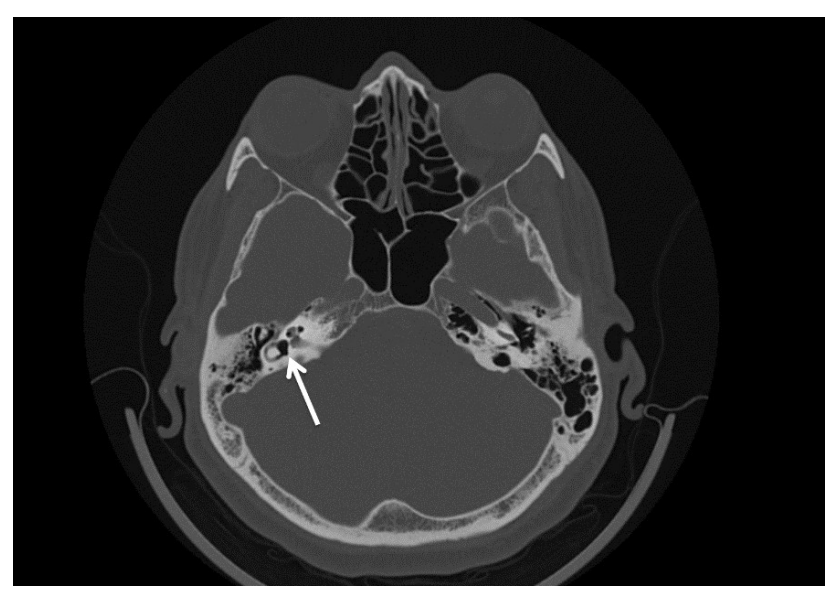

Fig. 3: An axial bone window CT-scan of the skull base illustrates a rightsided pneumolabyrinth (arrow).

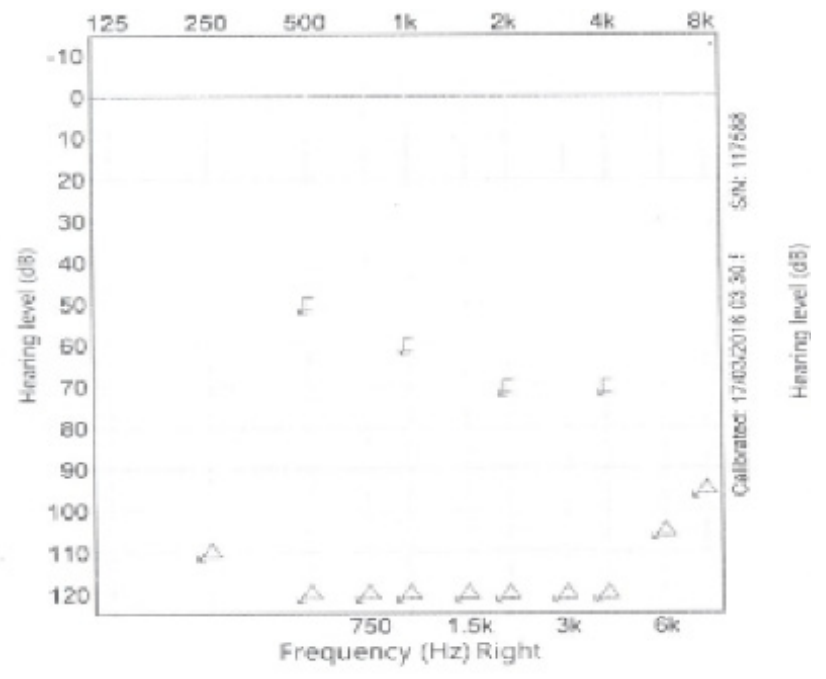

Fig. 1: Bone conduction values are displayed in brackets. Air conduction values are shown in triangles.

The patient was initially managed conservatively, with bed rest, oral corticosteroids, and antibiotics. No significant improvement of symptoms was observed after 1 week of conservative management and the patient continued to experience intense vertigo and imbalance. Therefore, surgical exploration, via a trans-canal approach, was undertaken. Intraoperatively, the ossicles were found to be intact and mobile. The stapes footplate was found in it is normal position, without subluxation into the vestibule. There was no evidence of perilymph leakage in the area of the oval or round windows. There was a non-displaced fracture line vertically crossing the medial wall of the middle ear through the round window niche and promontory, and bifurcating at the oval window. No perilymph leakage was observed around the fracture line. The fistula was sealed by applying a temporalis facial graft over the fracture line and around the stapes footplate.

Postoperatively, the patient was managed with bed rest and oral corticosteroids. The vertigo and imbalance improved markedly within 2 weeks after the surgery. However, the hearing thresholds of the right ear did not improve. Nine months after surgery, PTA showed no notable improvement in air or bone conduction thresholds 


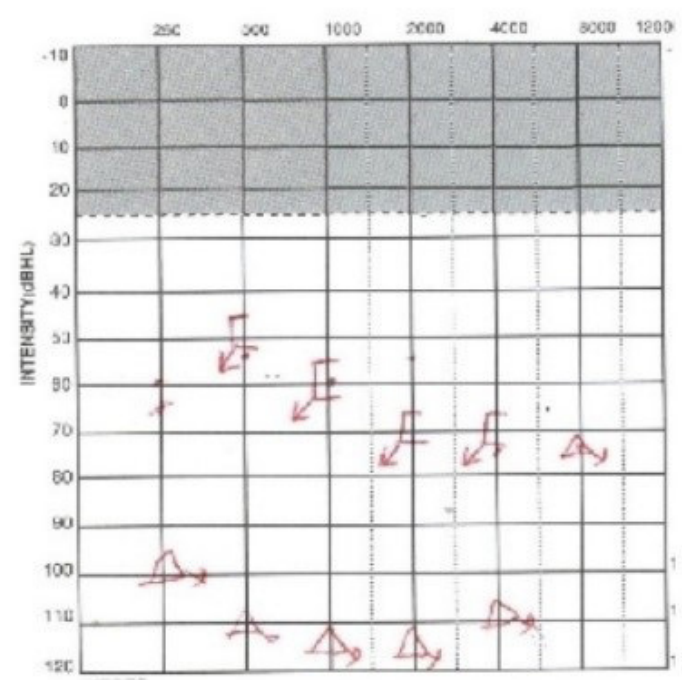

Fig. 2: Bone conduction values are displayed in brackets. Air conduction values are shown in triangles

\section{DISCUSSION}

Temporal bone fractures can be classified radiologically into otic capsule-sparing and otic capsule-violating fractures ${ }^{[1,7]}$ The rate of pneumolabyrinth is highest among cases of otic capsule-violating fractures, and nonexistent among cases of otic capsule-sparing fractures ${ }^{[8]}$ Air bubbles are most commonly found in the vestibule or SCCs, followed by pneumolabyrinth in the vestibule or SCC extending to the cochlea. Air confined only to the cochlea has seldom been reported. ${ }^{[2,8]}$ Accordingly, CT imaging is a valuable tool for diagnosing pneumolabyrinth. Since a temporal bone fracture might not be evident on the initial brain $\mathrm{CT}$, damage to the vestibular organs, cochlea, or ossicular chain cannot be eliminated. A CT examination should include high-resolution millimeter-scale slices directed towards the temporal bone ${ }^{[9]}$ In our patient, pneumolabyrinth might have been detected earlier with the use of a high-resolution, thin-slice CT scan aimed at the temporal bone.

Vestibular symptoms are more likely to resolve after the commencement of treatment. ${ }^{[6]}$ On the other hand, hearing recovery outcomes seem to be influenced by the following factors: the interval until surgery, the presence of stapes lesions, and air location, as observed on $\mathrm{CT}^{[2,10,11,12]}$ With a longer interval until surgery, hearing recovery is reduced: $54 \%$ of patients receiving surgery with an injury-to-surgery interval of less than 2 weeks demonstrated improved hearing levels, while hearing recovery was evident in only $25 \%$ of patients receiving surgery 2 or more weeks after the trauma ${ }^{[2]}$ In this study, our patient underwent surgery 4 weeks after the head injury; early explorative tympanotomy could benefit patients with a similar presentation. Second, stapes involvement was associated with a reduced hearing recovery ${ }^{[2,10]}$ Third, it has been proposed that the gas bubble location is correlated with the hearing prognosis.
Recovery of hearing was greatest in cases where the air was present within vestibular organs ${ }^{[8]}$ On the other hand, irreversible hearing loss was observed in cases where the air was present within the cochlea ${ }^{[11,12]}$ However, Choi et $a l .{ }^{[8]}$ suggested that hearing outcomes, in cases of traumatic pneumolabyrinth secondary to temporal bone fractures, are not influenced by the air location. Violation of the membranous labyrinth, mostly associated with otic capsule-violating fractures, were presumed to be the cause of the irreversible hearing loss, irrespective of the location of the air bubbles.

It should be noted that air and bone conduction hearing thresholds, before the commencement of treatment, failed to predict hearing outcomes. In a recent review by Hidaka et al., ${ }^{[2]}$ patients were categorized into four groups. Groups A and B were stratified by the average air conduction hearing level. Group A included 13 cases with an average air conduction hearing level of less than $65 \mathrm{~dB}$ HL. Of these 13 cases, six patients (46\%) improved, $31 \%$ deteriorated, and the remaining cases exhibited no change in hearing levels after treatment. Group B included cases with an average air conduction hearing level of $65 \mathrm{~dB}$ HL or greater. Of the 22 cases in group B, $41 \%$ of cases improved and deterioration was evident in $18 \%$ of cases. Similarly, groups C and D were categorized on the basis of average bone conduction hearing levels before starting management. The group with the better bone conduction hearing level failed to demonstrate superior hearing outcomes when compared with those of the other group.

Due to the limited number of cases reported in the current literature, guidelines for managing pneumolabyrinth are non-existent ${ }^{[14]}$ Traumatic pneumolabyrinth can be managed either conservatively or surgically, depending on the preference of the treating physician ${ }^{[13-14]}$ Although explorative tympanotomy seems to yield similar hearing outcomes as conservative management, worsening vestibular symptoms mandates that a surgical approach be taken $^{[2,13,14]}$ Recent work by Woo HJ et al. suggested that when combined pneumolabyrinth is treated conservatively, as in our patient, it can induce irreversible hearing loss ${ }^{[12]}$ In this patient, an active approach was undertaken, as the initial conservative approach was unsuccessful. His vertigo and imbalance were markedly improved postoperatively; however, no hearing recovery was evident on PTA.

\section{CONCLUSION:}

In the patient described here, poor hearing outcome could be due to the location of the air foci and the delay in medical and surgical interventions. Otic capsule-violating fractures might not be observed on brain CT scans commonly performed for head injury patients. Therefore, high-resolution and thin-slice CT scans directed towards the temporal bone is important in cases with suspected temporal bone fracture. 


\section{CONFLICT OF INTEREST}

There are no conflict of interest

\section{REFERENCES:}

1. Bacciu A, Vincenti V, Chandra Prasad S, Tonni D, Ventura E, Bacciu S, Pasanisi E. Pneumolabyrinth secondary to temporal bone fracture: a case report and review of the literature. International Medical Case Reports Journal.2014;7:127-31.

2. Hidaka H, Miyazaki M, Kawase T, Kobayashi T. Traumatic Pneumolabyrinth. Otology \& Neurotology. 2012;33(2):123-131.

3. Mafee M, Valvassori G, Kumar A, Yannias D, Marcus R. Pneumolabyrinth: a new radiologic sign for fracture of the stapes footplate. The American Journal of Otology. 1984;5(5):374-375.

4. Nurre J, Miller G, Ball J. Pneumolabyrinth as a late sequela of temporal bone fracture. The American journal of otology. 1988;9(6):489-493.

5. Hatano A, Rikitake $M$, Komori $M$, Irie $T$, Moriyama H. Traumatic perilymphatic fistula with the luxation of the stapes into the vestibule. Auris Nasus Larynx. 2009;36(4):474-478.

6. Mandalà M, Colletti L, Carner M, Barillari M, Cerini R, Mucelli R, Colletti V. Pneumolabyrinth and positional vertigo after stapedectomy. Auris Nasus Larynx. 2011;38(4):547-550.

7. Dahiya R, Keller J, Litofsky N, Bankey P, Bonassar L, Megerian C. Temporal Bone Fractures: Otic Capsule Sparing versus Otic Capsule Violating Clinical and Radiographic Considerations. The Journal of Trauma: Injury, Infection, and Critical Care. 1999;47(6):1079.
8. Choi H, Lee H, Lee J, Kim D, Hong S, Park B, Kim S, Kim J, Kim H. The Rates and Clinical Characteristics of Pneumolabyrinth in Temporal Bone Fracture. Otology \& Neurotology. 2015;36(6):1048-1053.

9. Achache M, Sanjuan Puchol M, Santini L, Lafont B, Cihanek M, Lavieille J, Devèze A. Late pneumolabyrinth after undiagnosed post-traumatic perilymphatic fistula. Case report illustrating the importance of systematic emergency management. European Annals of Otorhinolaryngology, Head and Neck Diseases. 2013;130(5):283-287.

10. Tsubota M, Shojaku H, Watanabe Y. Prognosis of inner ear function in pneumolabyrinth: case report and literature review. American Journal of Otolaryngology. 2009;30(6):423-426.

11. Rother T, Albrecht C, Issing P. Pneumolabyrinth after cochlear implantation in large vestibular aqueduct syndrome: a case report. American Journal of Otolaryngology. 2011;32(5):430-432.

12. Woo H, Song S, Kim Y, Bai C. Pneumolabyrinth Without Temporal Bone Fracture: Different Outcomes for Hearing Recovery. The Laryngoscope. 2008;118(8):1464-1466.

13. Yanagihara N, Nishioka I. Pneumolabyrinth in perilymphatic fistula: report of three cases. The American journal of otology. 1987;8(4):313-318.

14. Sarac S, Cengel S, Sennaroglu L. Pneumolabyrinth following traumatic luxation of the stapes into the vestibule. International Journal of Pediatric Otorhinolaryngology. 2006;70(1):159-161. 